\title{
Applied cognitive computing: challenges, approaches, and real-world experiences
}

\author{
Mauro Dragoni ${ }^{1} \cdot$ Marco Rospocher $^{1}$ (i)
}

Published online: 24 September 2018

(c) Springer-Verlag GmbH Germany, part of Springer Nature 2018

Cognitive computing investigates the development of selflearning systems that naturally interact with humans in complex environments and are capable to adapt to context and changes in language and meaning. These systems mimic the human mind processes, elaborating huge amounts of data, finding correlations, testing hypotheses, making predictions and inferences, drawing conclusions, and so on. This is an extremely interdisciplinary emerging research area, at the core of artificial intelligence, combining and complementing the scientific results from various disciplines, such as natural language processing, knowledge representation and reasoning, audio and video analysis, neuroscience, computer-human interaction.

This Special Issue focuses on works describing uses of cognitive computing techniques in concrete scenarios, highlighting why these techniques demonstrated their suitability and which are the challenges they solved. We have received nine submissions in response to our call for papers that were rigorously evaluated by a number of expert reviewers. At the end of the reviewing process, we were able to select four quality papers that showcase interesting concrete applications of cognitive computing techniques.

The first paper, "SocialLink: Exploiting Graph Embeddings to Link DBpedia Entities to Twitter Profiles" by Yaroslav Nechaev, Francesco Corcoglioniti, and Claudio Giuliano, presents a work for automatically matching, leveraging deep neural network-based techniques, Twitter profiles to corresponding entities in DBpedia, thus linking the Twitter social media world to the Linked Open Data cloud. This enables a two-way knowledge transfer between Twitter and DBpedia, fruitful for both social media analysis and knowledge extraction from social media content. The work extends a previous contribution of the authors, presented at the first

\footnotetext{
Marco Rospocher

rospocher@fbk.eu

Mauro Dragoni

dragoni@fbk.eu

1 Fondazione Bruno Kessler, Trento, Italy
}

edition of the Cognitive Computing special track [1] we organized at the 32nd edition of the ACM Symposium on Applied Computing, held in Marrakesh (Morocco).

The second paper, "Using Frame-Based Resources for Sentiment Analysis within the Financial Domain" by Mattia Atzeni, Amna Dridi, and Diego Reforgiato Recupero, investigates sentiment analysis in the financial domain. By leveraging frame-based ontological resources, the authors propose a supervised approach to identify bullish and bearish sentiments, associated with companies and stocks, in blogs and social networks. The conducted evaluation provides evidence of the benefit of embodying frame-based ontological resources in sentiment analysis methods.

The third paper, "Review-Aggregated Aspect-Based Sentiment Analysis with Ontology Features" by Sophie de Kok, Linda Punt, Rosita van den Puttelaar, Karoliina Ranta, Kim Schouten, and Flavius Frasincar, investigates aspect-based sentiment analysis for textual reviews. In particular, the approach employs ontology features to determine the sentiment value of a given pair of review and aspect. The conducted evaluation confirms the usefulness of ontology features to correctly determine the sentiment of review aspects. The work extends a previous contribution of the authors, presented at the second edition of the Cognitive Computing special track [2] we organized at the 33rd edition of the ACM Symposium on Applied Computing, held in Pau (France).

The fourth paper, "Exploring the Association between Mobility Behaviours and Academic Performances of Students: A Context aware Traj-Graph (CTG) Analysis" by Shreya Ghosh and Soumya K. Ghosh, analyzes the correlation between mobility information of students, collected for instance from GPS traces, and their academic performance. The authors propose a framework where mobility patterns of students are modeled using context aware trajgraph. In particular, the presented experimental evaluation, conducted on real data collected in an academic campus, shows that students' academic performance can be predicted from their movement behaviors. 
We would like to sincerely thank all the reviewers that contributed to the selection of the articles included in this Special Issue. A special thanks to the journal editors, Prof. Salvador García López and Prof. Francisco Herrera, for their availability in hosting this Special Issue in the Progress in Artificial Intelligence journal, and to Ms. Sharmila Prathaban, for her support in handling the submission process.

\section{References}

1. Dragoni, M., Rospocher, M.: Special track on cognitive computing. In: Proceedings of the 32nd Annual ACM Symposium on Applied Computing (SAC 2017), Marrakesh, Morocco, 4-6 April 2017, pp. 123-124, Editorial (2017)
2. Dragoni, M., Rospocher, M.: Special track on cognitive computing. In: Proceedings of the 33rd Annual ACM Symposium on Applied Computing (SAC 2018), Pau, France, 9-13 April 2018, Editorial (2018) 